\title{
Modelling of the anti-neutrino production and spectra from a Magnox reactor
}

\author{
Robert W Mills, David J Mountford, Jonathon P. Coleman, Carl Metelko, Matthew Murdoch, \\ and Yan-Jie Schnellbach
}

\begin{abstract}
The anti-neutrino source properties of a fission reactor are governed by the production and beta decay of the radionuclides present and the summation of their individual antineutrino spectra. The fission product radionuclide production changes during reactor operation and different fissioning species give rise to different product distributions. It is thus possible to determine some details of reactor operation, such as power, from the anti-neutrino emission to confirm safeguards records. Also according to some published calculations, it may be feasible to observe different anti-neutrino spectra depending on the fissile contents of the reactor fuel and thus determine the reactor's fissile material inventory during operation which could considerable improve safeguards. In mid-2014 the University of Liverpool deployed a prototype anti-neutrino detector at the Wylfa R1 station in Anglesey, United Kingdom based upon plastic scintillator technology developed for the T2K project. The deployment was used to develop the detector electronics and software until the reactor was finally shutdown in December 2015. To support the development of this detector technology for reactor monitoring and to understand its capabilities, the National Nuclear Laboratory modelled this graphite moderated and natural uranium fuelled reactor with existing codes used to support Magnox reactor operations and waste management. The 3D multi-physics code PANTHER was used to determine the individual powers of each fuel element $(8 \times 6152)$ during the year and a half period of monitoring based upon reactor records. The WIMS/TRAIL/FISPIN code route was then used to determine the radionuclide inventory of each nuclide on a daily basis in each element. These nuclide inventories were then used with the BTSPEC code to determine the anti-neutrino spectra and source strength using JEFF-3.1.1 data. Finally the anti-neutrino source from the reactor for each day during the year and a half of monitored reactor operation was calculated. The results of the preliminary calculations are shown and limitations in the methods and data discussed.
\end{abstract}

Index Terms-Antineutrino; Reactor spectrum; Nuclear reactor; Fission Product Yields; Nuclear safeguards

The work of the UK National Nuclear Laboratory (NNL) authors was supported by the NNL's Innovation Research and Development programme. The University of Liverpool authors acknowledge the support of the STFC and the Royal Society.

R. W. Mills is with the National Nuclear Laboratory, Central Laboratory, Sellafield, Seascale, Cumbria, United Kingdom. (e-mail: robert.w.mills@ nnl.co.uk).

D. J. Mountford was with the National Nuclear Laboratory, Central Laboratory, Sellafield, Seascale, Cumbria, United Kingdom during this work.

J. P. Coleman, C. Metelko, M. Murdoch, and Y. Schnellbach are with the University of Liverpool, Liverpool L69 7ZE, United Kingdom.

\section{INTRODUCTION}

$T$ HE anti-neutrinos emission from fission has been suggested as a way to monitor nuclear reactors for some time as they have extremely low rates of interaction with matter and thus are considered impossible to shield their emission from reactors. However, this extremely low interaction rate makes the detection of anti-neutrinos very difficult.

There are several steps that are necessary for such reactor monitoring to become a practical technology. Firstly, a detector must be constructed that can uniquely identify reactor anti-neutrinos considering radiations of other types such as reactor neutrons and gamma-rays, and naturally occurring radiations from the environment such cosmic rays, neutrons, gamma-rays and other neutrino sources (including antineutrinos from the beta decay of naturally occurring radioisotopes). Secondly, the detector must be easily used in the area near the nuclear reactor. Nuclear Safety on such sites requires that any equipment must not introduce risks to the reactor or its staff. Specifically, the equipment should not introduce extra chemical, physical, fire or radiation risks, nor should it need to be incorporated into existing safety systems or have significant power requirements. Due to security access issues the equipment should be able to be left as a standalone unit not needing many maintenance visits with most contact being via remote access methods, whilst allowing interference with the equipment to be easily detected remotely. Finally, the detector should easily supply information on the reactor without the need for complex analysis. To ensure that the equipment is useful it must be demonstrated that the detector can function on a nuclear site and that the reported information obtained from the detector be verified against plant records by carrying out experiments on well characterized reactors.

This paper reports work considering the University of Liverpool anti-neutrino detector that was placed near the Wylfa reactor building for 18 months, prior to the last Wylfa reactor core being shut down.

\section{THE UNIVERSITY OF LIVERPOOL DETECTOR}

The detector, now called VIDARR, is about $1 \mathrm{~m}^{3}$ of plastic scintillator detector using Si photomultipliers. It is based upon the technology of the T2K neutrino experiment, which works by using the inverse beta decay reaction on hydrogen in the 


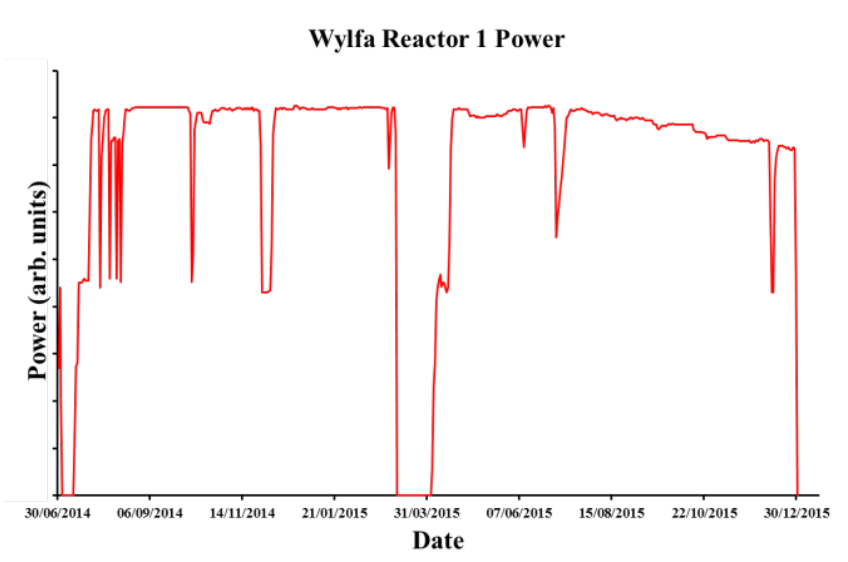

Fig. 1. Wylfa reactor 1 power during detector deployment showing both two periods of start-up and shutdown with periods of low power to replace or remove or shuffle fuel within the core.

plastic $\left(\overline{v_{e}}+p \rightarrow e^{+}+n\right)$. The positron produces two annihilation photons and the neutron is scattered around the volume until it is captured by the trace of gadolinium introduced into the plastic. The neutron capture reaction results in a delayed radiation signature. From the energies of the two annihilation photons it is possible to trigger on an antineutrino capture event and from the radiation produced by the neutron capture reaction to identify the events as an antineutrino capture and determine the energy of the anti-neutrino. The detector events from VIDARR were processed and stored on a computer within the instrumentation rack, which could be accessed via a phone connection.

As with all inverse beta decay detectors, the threshold of the anti-neutrino reaction on hydrogen is about $1.8 \mathrm{MeV}$ and then the cross-section rapidly rises. However, the anti-neutrino spectrum above $1.8 \mathrm{MeV}$ rapidly reduces with increasing antineutrino energy. Thus the probability of detection is zero below $1.8 \mathrm{MeV}$ and rises rapidly above this, peaking around 4 $\mathrm{MeV}$ before rapidly declining at higher energies.

The detector was built at the University of Liverpool and installed in a refrigerated $20 \mathrm{ft}$ transport container. The stable, lower than ambient temperature, in the container helped to reduce the electronic noise from the photomultipliers.

In the middle of 2014 the container was transported to the Wylfa reactor site in North Wales and installed in a layby on a site road at about $60 \mathrm{~m}$ from the centre of the operating Magnox reactor. The instrumentation and cooling equipment was powered by a single cable from a convenient power socket and the equipment was connected via a phone line for remote access to the computer.

The above ground installation of the detector leads to a high cosmic ray background that complicated the identification of anti-neutrino events detected.

\section{REACTOR OPERATION DURING DEPLOYMENT}

The Wylfa reactor complex consists of two reactors in a single reactor building linked to an adjacent turbine building which generates the electrical power. Reactors 1 and 2 were built from 1963 and became operational in 1971. They were

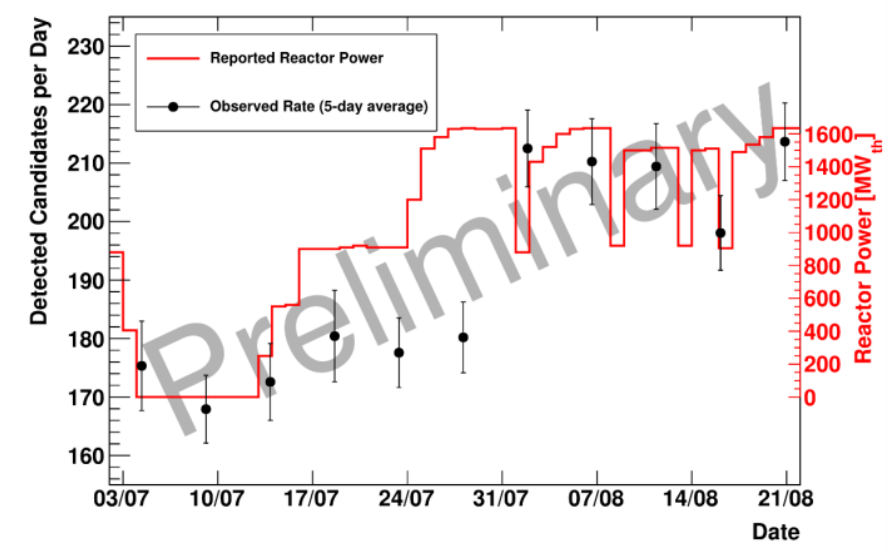

Fig. 2. Wylfa reactor 1 power during the July 2014 start-up in MW thermal and the 5-day averaged event detection rate (per day).

initially each rated at $570 \mathrm{MW}$ electrical, but during operation this was reduced to $490 \mathrm{MW}$ electrical. The reactor 2 was shut down in 2012, but reactor 1 operated until December 2015, giving about 18 months of operation during which the detector was deployed.

Each of the two Wylfa reactor cores consists of a graphite moderator containing 6156 fuel channels into to each of which can be inserted up to 8 Magnox fuel elements. Each element consists of a uranium metal cylinder surround by a Magnesium alloy cladding (Magnox ${ }^{\mathrm{TM}}$ ) with end-fittings joining the elements in each channel. The uranium is natural or 0.8 wt. $\%{ }^{235} \mathrm{U}$ w.r.t. $\mathrm{U}$ with each element containing about $12 \mathrm{~kg}$ of uranium when installed. The total core loading was approximately 593 metric tons of uranium. Wylfa is unusual for Magnox reactors in that on-power refueling was regularly carried out but this required the reactor power to be significantly reduced during the fuel movements.

The reactor power during the period with the detector installed is shown in figure 1 .

\section{PRELIMINARY RESULTS FROM THE DETECTOR}

During the initial period of operation, the detector identified about 170 events per day during shutdown, but this rose to about 210 events during full power operation. Some preliminary results are shown in figure 2. These preliminary results show that the detector can be used to identify reactor power, but that improvements are required in the analysis of the detector response to cosmic rays and other background radiations to improve the identification of anti-neutrinos.

\section{CALCULATIONS METHODOLOGY FOR ANTI-NEUTRINO EMISSION ESTIMATION}

The full operational data of the Wylfa reactor 1 was obtained by NNL including the full power history and the fuel element's irradiation ( $\mathrm{MWd} / \mathrm{t})$, power rating $(\mathrm{MW} / \mathrm{t})$, initial enrichment and loading dates for the whole core during the last 18 months of operation. This was calculated using the PANTHER whole core modelling code. The power and shutdown history was then used to estimate the full radionuclide inventory in each of the 49248 fuel elements 
using the UK spent fuel inventory code FISPIN for each day of the reactor operation and shutdown. This gave the reactor's radionuclide inventory and the beta decay rate of all the isotopes present.

The beta spectra were then calculated using the BTSPEC code with the latest JEFF-3.1.1 decay data for all 670 radionuclides with sufficient information in this library. The code was modified to use the end-point energies of each transition to estimate the corresponding anti-neutrino spectra. It should be noted that these 670 nuclides represent about $90 \%$ of the beta decays in the fuel during operation rising to $100 \%$ during shutdowns, but that without more information on the short-lived radionuclides and their beta decay properties that it is not possible to estimate their anti-neutrino spectra. In this work the beta particle and anti-neutrino spectra were generated in $10 \mathrm{keV}$ bins between 0 and $15 \mathrm{MeV}$.

By combining the spectra data and the radionuclide activities for each rod it is possible to estimate the anti-neutrino emission and spectra from the reactor as a 3 dimensional antineutron source.

Figure 3 shows the anti-neutrino flux with energy on-power and over several days after shutdown. This shows that the antineutrino flux detectable by the inverse beta decay method (above the $1.8 \mathrm{MeV}$ reaction threshold) quickly falls off after shutdown. This suggests that spent fuel storage near to the reactor will not interfere with the detector.

The anti-neutrino flux can also be calculated using FISPIN from fission for the separate fissionable materials present, principally ${ }^{235} \mathrm{U},{ }^{239} \mathrm{Pu},{ }^{241} \mathrm{Pu}$ and ${ }^{238} \mathrm{U}$. These results were compared with the experimental data calculated from experiments on beta decay spectra measured from pulsed irradiations on samples of pure fissile species. The experiment to calculation ratios for ${ }^{235} \mathrm{U},{ }^{238} \mathrm{U},{ }^{239} \mathrm{Pu}$ and ${ }^{241} \mathrm{Pu}$ are shown in figure 4. These results show quite good agreement with measured values for ${ }^{235} \mathrm{U}$ and ${ }^{239} \mathrm{Pu}$ above $3 \mathrm{MeV}$ being within the one standard deviation uncertainties on the anti-neutrino spectra calculated from the fission product yield uncertainties (shown in the graphs by the blue lines). The agreement for ${ }^{238} \mathrm{U}$ and ${ }^{241} \mathrm{Pu}$, although poorer, is still within a range of the ratio between 1.4 and 0.5 . This suggests that the result of using this calculation methodology is probably accurate to 10 $20 \%$ for thermal reactor fuels where fission of ${ }^{235} \mathrm{U}$ and ${ }^{239} \mathrm{Pu}$ dominate.

\section{CONCLUSIONS}

The initial result of this work has suggested that the FISPIN calculations used to generate inventories for all 49248 rods in the core during each day of reactor operation with irradiation/cooling history should give an estimate of the antineutrino flux and spectra to within $20 \%$.

It is intended to use the results of the Wylfa PANTHER and FISPIN calculations combined with the bespoke anti-neutrino source (number and spectra) calculation code to generate antineutrino spectra for each rod in the core during each day of the monitored period. These will then be used to produce an antineutrino flux with direction information over the detector location to facilitate GEANT-4 modelling of the detector to help understand its response properties and what the detector can achieve in determining the reactor state.

The study of the underlying nuclear data used in the calculations will also continue to consider if better nuclear data and modelling of the beta decay will improve the estimation of the anti-neutrino source term.

\section{ACKNOWLEDGEMENTS}

The authors wish to thank G. Davies, A. Roberts and A. Tobias of Magnox Ltd, which operated the Wylfa reactor, for information on the reactor and many helpful discussions on its operation.

\section{Expected Anti-neutrinos per Energy}

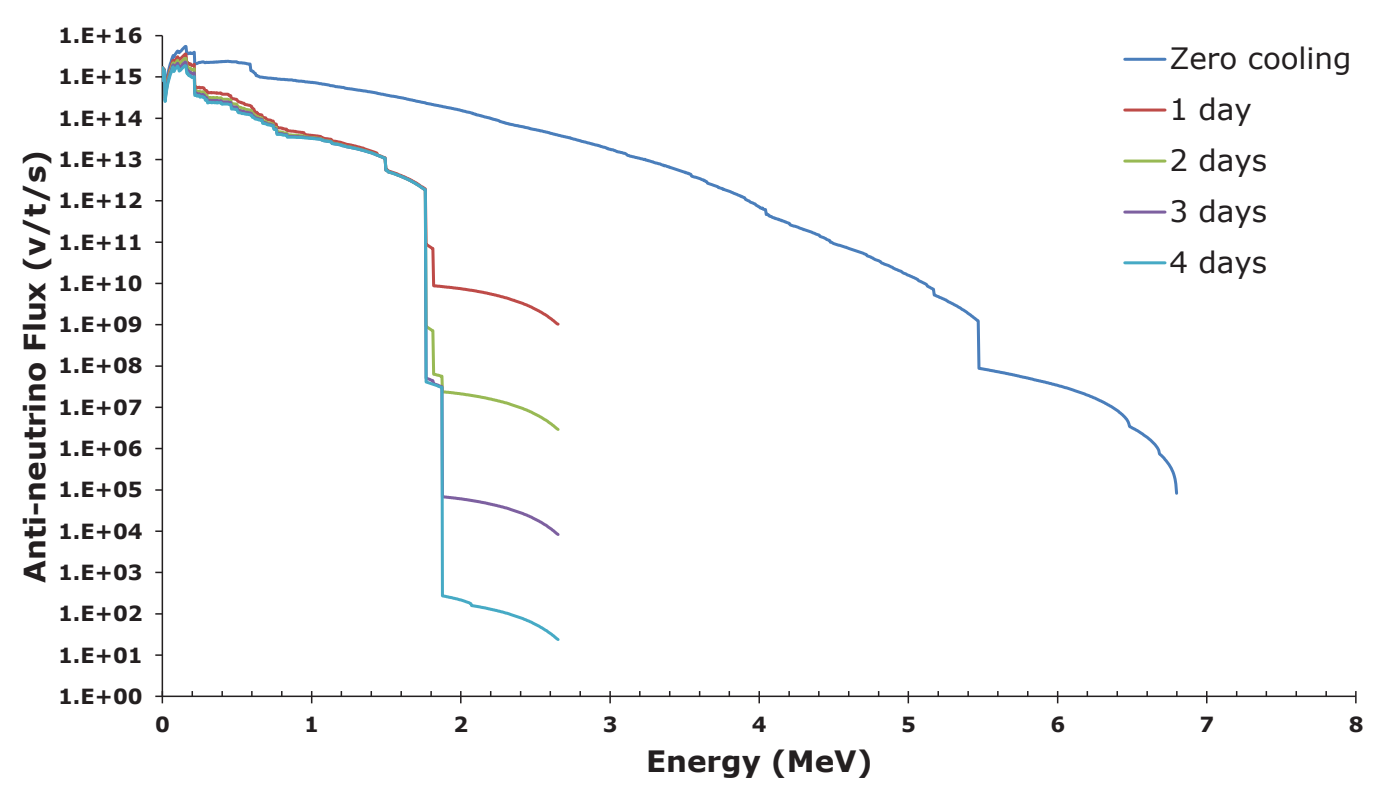

Fig. 3. Estimation of the anti-neutrino spectra per metric ton of uranium in the Wylfa reactor at the moment of shutdown and for the following 4 days using FISPIN. 

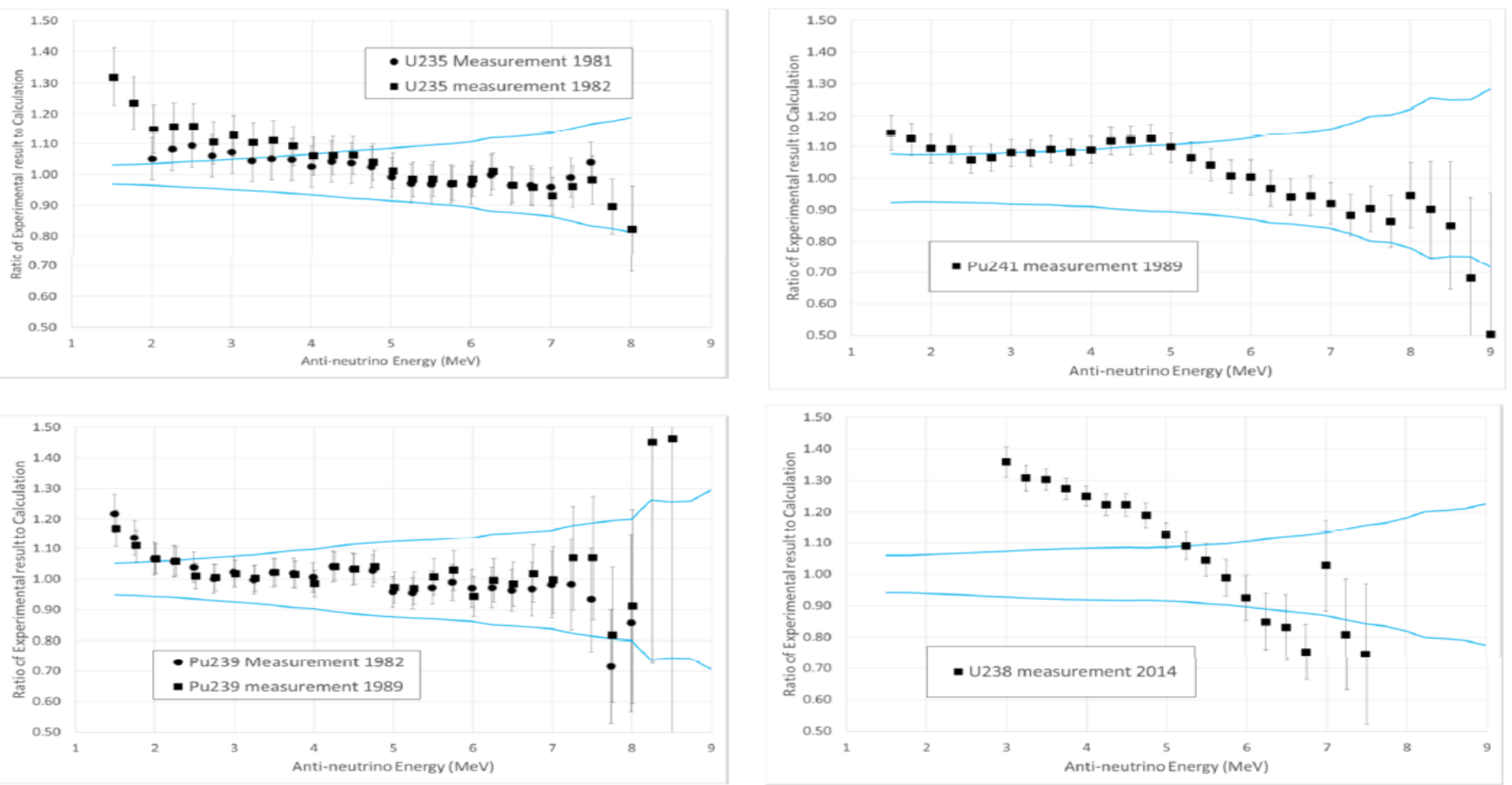

Fig. 4. Comparison of the estimated anti-neutrino flux spectra against the available experimental measurements by plotting experiment over calculation ratios for ${ }^{235} \mathrm{U}$, ${ }^{238} \mathrm{U}$, ${ }^{239} \mathrm{Pu}$ and ${ }^{241} \mathrm{Pu}$. The blue lines show the uncertainties on the calculation from fission product yields.

\section{REFERENCES}

[1] Abe, Y., dos Anjos, J.C. et al. "The Double Chooz collaboration", J. High Energ. Phys. (2014) 86. doi:10.1007/JHEP10(2014)086

[2] An, F.P., Balantekin, A.B., Band, H.R., Bishai, M., Blyth, S., Butorov, I., Cao, D., (...), Zou, J.H. "Measurement of the reactor antineutrino flux and spectrum at Daya Bay" (2016) Physical Review Letters, 116 (6), art. no. 061801

[3] Choi, J.H., Choi, W.Q., Choi, Y., Jang, H.I., Jang, J.S., Jeon, E.J., Joo, K.K., Yu, I. "Observation of Energy and Baseline Dependent Reactor Antineutrino Disappearance in the RENO Experiment " (2016) Physical Review Letters, 116 (21), art. no. 211801

[4] Feilitzsch, F. von, Hahn A.A. and Schreckenbach, K., "Experimental beta-spectra from ${ }^{239} \mathrm{Pu}$ and ${ }^{235} \mathrm{U}$ thermal neutron fission products and their correlated antineutrino spectra" (1982) Physics Letters, Volume 118B, number 1, 2,3

[5] Haag, N., Gütlein, A., Hofmann, M., Oberauer, L., Potzel, W., Schreckenbach, K., Wagner, F.M. "Experimental determination of the antineutrino spectrum of the fission Products of U 238" (2014) Physical Review Letters, 112 (12)

[6] Hahn A.A., Schreckenbach, K., Gelletly, W., Feilitzsch, F. von, Colvin, G. and Krusche, B. "ANTINEUTRINO SPECTRA FROM ${ }^{241} \mathrm{Pu}$ AND ${ }^{239} \mathrm{Pu}$ THERMAL NEUTRON FISSION PRODUCTS" (1989) Physics Letters B Volume 218, number 3

[7] Huber, P. "NEOS Data and the Origin of the $5 \mathrm{MeV}$ Bump in the Reactor Antineutrino Spectrum" (2017) Physical Review Letters, 118 (4), art. no. 042502.

[8] P.K. Hutt, N. Gaines, M.J. Halsall, M. McEllin, R.J. White, " The UK core performance code package", Nuclear Energy, British Nuclear Energy Society, 30 (1991), p. 291
[9] FISPIN for Nuclide Inventory Calculations: Introductory Guide for Version 10, 2007. Report ANSWERS/FISPIN/REPORT/001 published by Serco Assurance Ltd.

[10] Kellett, M. A., O. Bersillon, and R. W. Mills. "The JEFF3.1/-3.1.1 radioactive decay data and fission yields sublibraries." JEFF report 20 (2009)

[11] Mills, R.W. "Uncertainty propagation of fission product yield data in spent fuel inventory calculations" (2014) Nuclear Data Sheets, 118 (1), pp. 484-487

[12] Mougeot, X. "Systematic comparison of beta spectra calculations using improved analytical screening correction with experimental shape factors" (2016) Applied Radiation and Isotopes, 109, pp. 177-182.

[13] Mueller, Th. A., Lhuillier, D., Fallot, M., Letourneau, A., Cormon, S., Fechner, M., Giot, L., Lasserre, T. , Martino, J., Mention, G., Porta, A. and Yermia F. "Improved predictions of reactor antineutrino spectra", (2011) Phys. Rev. C 83, 054615

[14] Oralbaev, A., Skorokhvatov, M., Titov, O. "The inverse beta decay: A study of cross section" (2016) Journal of Physics: Conference Series, 675 (1), art. no. 012003, p. 37.

[15] Schreckenbach, K., Faust, H.R., Feilitzsch, F. von, Hahn, A.A.,Hawrkamp, K. and Vuilleumier J.L. "Absolute measurement of the beta spectrum from $235 \mathrm{U}$ fission as a basis for reactor antineutrino experiments" (1981) PHYSICS LETTERS Volume 99B, number 3

[16] Seo, S.-H "New results from RENO and the $5 \mathrm{MeV}$ excess" (2015) AIP Conference Proceedings, 1666, art. no. 080002.ISBN: 978-073541313-9

[17] Tobias, A. "A Retrieval System for Spectral Data from ENDF/B Format Decay Data Files", (1981) CEGB report $\mathrm{RD} / \mathrm{B} / 5170 \mathrm{~N} 81$ 